\title{
La enseñanza de la matemática en la carrera de Microbiología Clínica e Industrial durante la pandemia de la COVID-19: herramientas tecnológicas y educación con el enfoque: Ciencia, Tecnología, Sociedad (CTS)
}

\author{
Teaching Mathematics in the career of Clinical and Industrial Microbiology \\ during the COVID-19 pandemic: technology tools and education with the \\ focus: Science, Technology, Society (STS)
}

Lampert, Damián Alberto; Porro, Silvia

\section{DamiánAlbertoLampert \\ dlampert@fcv.unlp.edu.ar \\ Departamento de Ciencias Básicas, cursos de Matemática I y II. Carrera de Microbiología Clínica e Industrial. Facultad de Ciencias Veterinarias. \\ Universidad Nacional de La Plata, Argentina \\ Departamento de Ciencia y Tecnología, Universidad Nacional de Quilmes, Argentina \\ CONICET, Argentina}

\section{Silvia Porro}

Departamento de Ciencia y Tecnología, Universidad Nacional de Quilmes, Argentina

\section{ANALECTA VETERINARIA}

Universidad Nacional de La Plata, Argentina

ISSN: $1514-2590$

Periodicidad: Semestral

vol.41, núm.1,e055, 2021

analecta@fcv.unlp.edu.ar

Recepción: 06 Mayo 2021

Revisado: 31 Mayo 2021

Aprobación: 25 Junio 2021

URL:

http://portal.amelica.org/ameli/jatsRepo/25/2519840 o3/ index.html

DOI:https://doi.org/10.24215/15142590eo55
Resumen: Se presenta una investigación cualitativa, de carácter exploratoria y reflexiva, sobre la enseñanza de la matemática durante la pandemia de COVID-19, a partir de la utilización de diferentes herramientas tecnológicas y del abordaje del enfoque Ciencia, Tecnología, Sociedad (CTS). Se analizan las ventajas y desventajas de diferentes herramientas, tales como plataformas web, aplicaciones de videoconferencias y tabletas gráficas, para tratar de resolver problemas, de manera sincrónica, durante las clases virtuales. Enseñar matemática a partir del enfoque CTS, permite contextualizar los contenidos disciplinares en relación con temas de sociología e historia de la ciencia, analizando, por ejemplo, el desarrollo de una enfermedad.

Palabras clave: matemática, educación CTS, herramientas tecnológicas, pandemia.

Abstract: A qualitative, exploratory, and reflective research on the teaching of mathematics during the COVID-19 pandemic based on the use of different technological tools with the Science, Technology, Society (STS) approach is presented. The advantages and disadvantages of different tools, such as web platforms, videoconferencing applications and graphic tablets, are analyzed for problem solving during synchronous virtual classes. On the other hand, teaching mathematics from the STS approach allows contextualizing the disciplinary contents in relation to topics of sociology and history of science analyzing, for example, the development of a disease.

Keywords: mathematics, STS education, technological tools, pandemic. 


\section{Introducción}

La realización de ejercicios prácticos es una de las modalidades convencionales en la enseñanza de la matemática, teniendo en cuenta la naturaleza de la asignatura. Sin embargo, a partir de la cuarta y quinta revolución tecnológica, el uso de las Tecnologías de la Información y la Comunicación (TIC) ha modificado las prácticas educativas (Díaz Dávila et al., 2020). La incorporación de recursos como videos y aulas virtuales ofrece variedad, facilita la comprensión, favorece la simulación y sustenta el aprendizaje asincrónico (Díaz Dávila et al, 2020). Asimismo, la utilización de diferentes tipos de software, como el GeoGebra, permite una mayor interpretación del estudiantado en la lectura de gráficos y funciones, brindándole herramientas para llegar a una respuesta determinada (Álvarez \& Murúa, 2020). En palabras de Ascheri y Pizarro (2006): "Es importante que el software contemple no solamente las prácticas, sino que proporcione al estudiante ayuda en la solución de los problemas y brinde una retroinformación completa".

Durante el año 2020, la pandemia causada por el virus SARS-CoV-2 ha impulsado un cambio rotundo en la enseñanza de todos los niveles educativos en diferentes países (Cabezas Rosa, 2020; García-Planas \& Taberna Torres, 2021; Hidalgo-Moncada et al., 2020; Ortiz-Buitrago \& Sánchez, 2020; Rojas Maldonado \& Toscano Galeana, 2021). En Argentina, como consecuencia del "Aislamiento Social, Preventivo y Obligatorio", todos los niveles educativos han realizado un gran esfuerzo para continuar con la calidad educativa de forma virtual (Baumann, 2021; Bonservizi \& Sgreccia, 2021; Di Domenicantonio \& Langoni, 2021; Diaz Dávila et al., 2020; Lescano et al., 2021).

\begin{tabular}{|c|c|}
\hline Matemática I & Matemática II \\
\hline $\begin{array}{l}\text { Nociones de lógica. Números reales: } \\
\text { propiedades algebraicas y de orden. Ecua- } \\
\text { ciones e Inecuaciones donde interviene el } \\
\text { valor absoluto de un número real. Cálculo } \\
\text { de números aproximados. } \\
\text { Funciones: gráfica y desplazamientos. } \\
\text { Composición de funciones. Funciones } \\
\text { subreyectivas, inyectivas y biyectivas. } \\
\text { Función inversa. Funciones polinómicas y } \\
\text { racionales. Funciones exponencial y } \\
\text { logarítmica. Funciones trigonométricas. }\end{array}$ & $\begin{array}{l}\text { Nociones sobre sistemas de ecuaciones } \\
\text { algebraicas lineales. Vectores, operacio- } \\
\text { nes. Matrices, operaciones. Nociones } \\
\text { sobre determinantes. Complemento sobre } \\
\text { gráfica de funciones elementales. Deri- } \\
\text { vada de una función de una variable real. } \\
\text { Diferencial. Estudio de gráficos. Integra- } \\
\text { les de funciones continuas. Funciones } \\
\text { limitativas. Funciones de varias variables. } \\
\text { Derivadas parciales. Ecuaciones diferen- } \\
\text { ciales con variables separables. Aplica- } \\
\text { ción a situaciones concretas de problemas } \\
\text { microbiológicos. }\end{array}$ \\
\hline
\end{tabular}

Tabla 1. Contenidos mínimos de Matemática I y Matemática II (Ordenanza 282 de la Carrera de Microbiología Clínica e Industrial, Facultad de Ciencias Veterinarias, UNLP, 2013).

En la carrera Microbiología Clínica e Industrial de la Facultad de Ciencias Veterinarias, de la Universidad Nacional de La Plata (UNLP), se incluyen los cursos de Matemática I (en el primer año) y Matemática II (en el segundo año). Los contenidos de ambos cursos se asocian con álgebra, geometría analítica y cálculo numérico. En la Tabla 1 se presentan los contenidos mínimos. 
Esta variedad de temas requiere de la utilización de diferentes herramientas tecnológicas que permita no eximir a la matemática del proceso concreto, gráfico y abstracto (Sánchez Pachas, 2020). Más allá de las herramientas tecnológicas a utilizar, en el contexto de la COVID-19, se debe enseñar la matemática que explique fenómenos epidemiológicos al tratarse de una carrera de microbiología y en la que los contenidos mínimos detallan las aplicaciones en microbiología. Por ejemplo: "Los estudiantes saben que el incremento de los contagios se expresa a través de un crecimiento exponencial, una simulación o proyección. Esta se convierte en un punto de partida para comprender el porqué del algebra que muchas veces, por su abstracción, no es visible a los ojos de los estudiantes" (Sánchez Pachas, 2020).

En este sentido, es importante resaltar el papel de la educación con enfoque Ciencia, Tecnología, Sociedad (CTS). La enseñanza de las ciencias con enfoque CTS permite la construcción del conocimiento científico y el desarrollo de actitudes y capacidades para el pensamiento (Tenreiro-Vieira \& Vieira, 2016). De esta forma, la educación CTS permite acercar al estudiantado a la realidad que lo rodea, incorporando contenidos de "Naturaleza de la Ciencia y la Tecnología" (NdCyT). La NdCyT hace referencia a metaconocimientos acerca del funcionamiento de la ciencia y la tecnología desde diversas miradas históricas, filosóficas y sociológicas.

La realización de secuencias didácticas en la dimensión CTS sobre asuntos sociocientíficos implica un mayor entendimiento de la NdCyT y sus relaciones con la sociedad (Zenteno-Mendoza \& Garritz, 2010). Como algunos ejemplos de universidades, se puede mencionar la inclusión de temas de Historia de la Química en los relacionados a la Química Inorgánica, en carreras de Ingeniería en Alimentos y Licenciatura en Biotecnología (Porro \& Roncaglia, 2016) y la inclusión de temas de género en la Licenciatura en Criminología y Ciencias (Carp et al., 2016).

Para las carreras de Ciencias Veterinarias e Ingeniería en Alimentos, Lampert y Porro (2019) y Lampert et al. (2020) propusieron una unidad didáctica sobre zoonosis y enfermedades transmitidas por alimentos en granjas educativas para las asignaturas Preservación de Alimentos, en el caso de Ingeniería, e Inmunología Animal Aplicada, para el caso de Medicina Veterinaria.

Teniendo en cuenta el contexto social mencionado, el enfoque CTS y las herramientas tecnológicas para la enseñanza de las matemáticas, este artículo pretende responder las siguientes preguntas:

- ¿Qué herramientas tecnológicas fueron las utilizadas en el dictado de Matemática I y de Matemática II durante la pandemia de la COVID-19?

- ¿Cómo se implementó el enfoque CTS en los cursos de Matemática I y II?

- ¿Cuáles fueron los resultados en relación con el porcentaje de estudiantado promocionado/aprobado sin promoción/abandonante en ambos cursos?

\section{Materiales y métodos}

Para responder las preguntas que guían este artículo, se llevó a cabo una investigación cualitativa y exploratoria de carácter reflexivo, en conjunto con un análisis cuantitativo, con el fin de comprender la relación entre las herramientas tecnológicas aplicadas y el enfoque utilizado para la enseñanza de la matemática con los resultados obtenidos en relación con el porcentaje de aprobación y deserción. Para ello, se trabajó con los cursos de Matemática I (41 estudiantes inscriptos/as) y Matemática II (10 estudiantes inscriptos/as) del ciclo lectivo 2020. Los cursos estaban 
compuestos por tres docentes (una persona cumple funciones de coordinación). Por tal motivo, la inclusión de distintas técnicas en la enseñanza de la matemática es fundamental, al igual que su utilización para fomentar el aprendizaje colaborativo del estudiantado.

\section{Resultados}

\section{Primera pregunta formulada}

Para responder la primera pregunta que hace referencia a las herramientas utilizadas, sehizo uso delos siguientes recursos:

\section{Plataforma Moodle}

La plataforma Moodle es un entorno virtual de enseñanza y aprendizaje que se utilizó como el espacio de intercambio y comunicación con el estudiantado. En la misma se presenta una serie de recursos que permiten llevar a cabo múltiples actividades y fomentar la comunicación asincrónica con el estudiantado.

En este espacio virtual se ponía a disposición del estudiantado el material, apuntes, guías y videos explicativos y se habilitaban foros de intercambio para cada recurso, a fin de fomentar el aprendizaje colaborativo(Figura 1). Elforopermitía que el estudiantado trabajara de forma asincrónica y colaborativa en la resolución de problemas y en el planteo de dudas de diferentes ejercicios, de forma que se generara una comunicación bidireccional, sin barreras, entre el tiempo y el espacio (Valarezo Neira, 2021). Esta herramienta permitía fomentar el seguimiento en la resolución de los diferentes problemas matemáticos, como así también, verificar y corregir los errores que podían presentar los ejercicios.

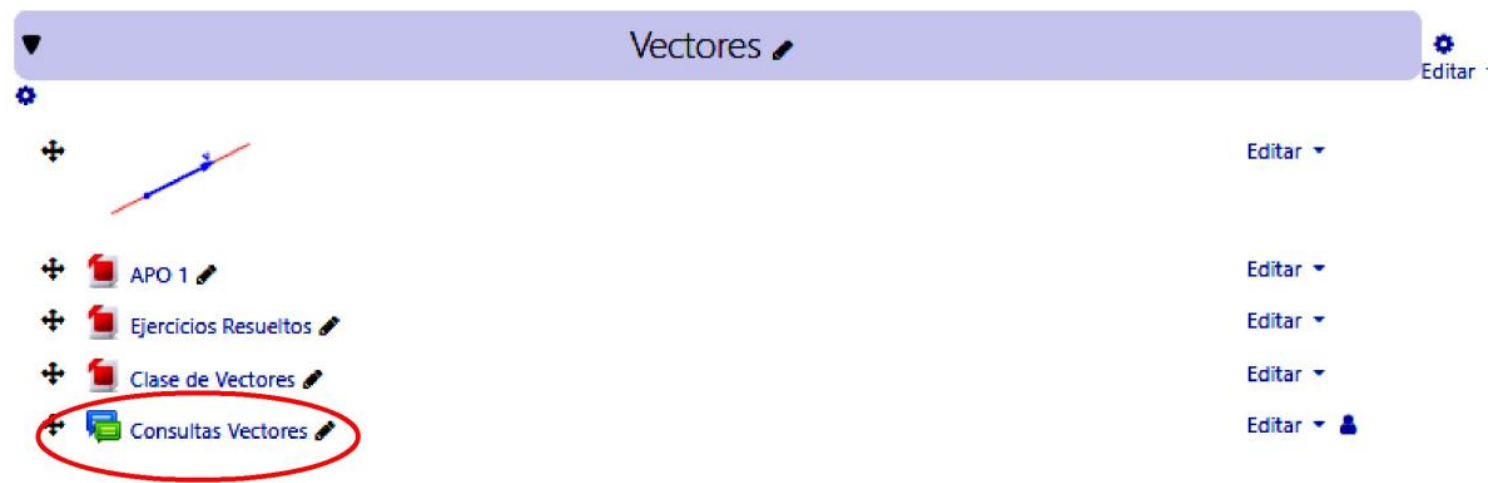

Figura 1. Imagen de la plataforma Moodle de Matemática II con un foro de trabajo colaborativo.

\section{Zoom}

Zoom es una aplicación que permite un sistema de videoconferencias. La selección de esta plataforma se utilizó debido al pedido del estudiantado de contar con las clases grabadas para aquellas personas que no podían asistir por cuestiones técnicas, como falta de internet, cortes de luz, etc. La desventaja es que, si no se posee una cuenta corporativa, el tiempo de la reunión es de 40 minutos. Ese corte permite tomar recreos y, además, que no se haga tan pesado el archivo para cargarlo a la plataforma. 


\section{Tableta gráfica}

Una de las grandes herramientas para la enseñanza de la matemática es el uso del pizarrón. A diferencia de otras asignaturas en las que tal vez pueden brindarse las clases con presentaciones o de forma dialogada, los contenidos mínimos de matemática para la carrera requieren de la resolución de problemas y demostraciones de propiedades. Por tal motivo, en un primer momento, se había planteado la posibilidad de utilizar un pizarrón con fibrón en el domicilio del profesorado. Sin embargo, la mayor desventaja es que el estudiantado no contaría con un apunte y hay quienes utilizan el teléfono celular para visualizar la clase, por lo que no podrían tomar apuntes de forma sincrónica. Por tal motivo, se utilizó una tableta gráfica. La misma es un dispositivo que se conecta a la computadora y permite al profesorado desarrollar la clase sobre un documento (como un procesador de texto) y, de esta forma, que al estudiantado se le proporcione, alfinalizar la clase, ese apunte (Figura 2).

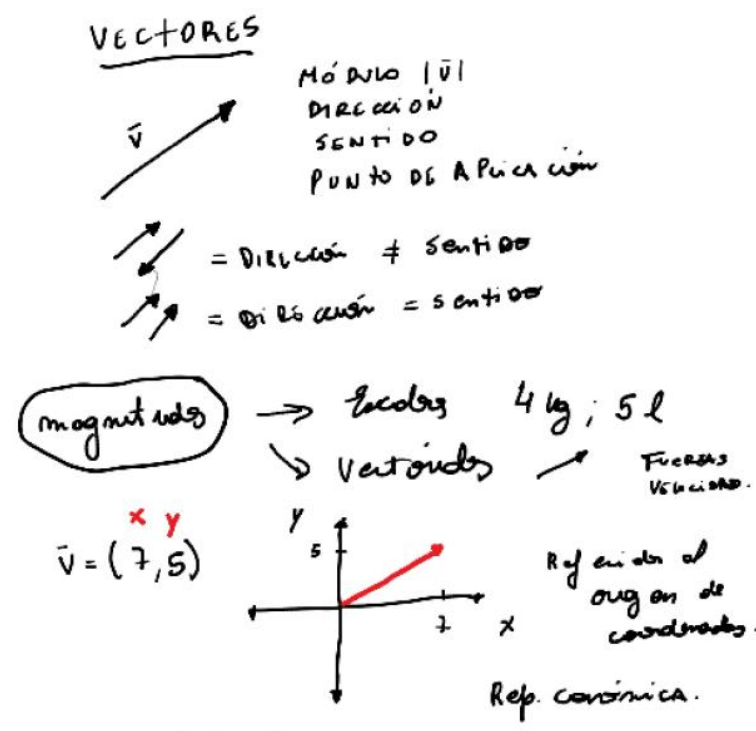

Figura 2: Ejemplo del uso de la tableta gráfica.

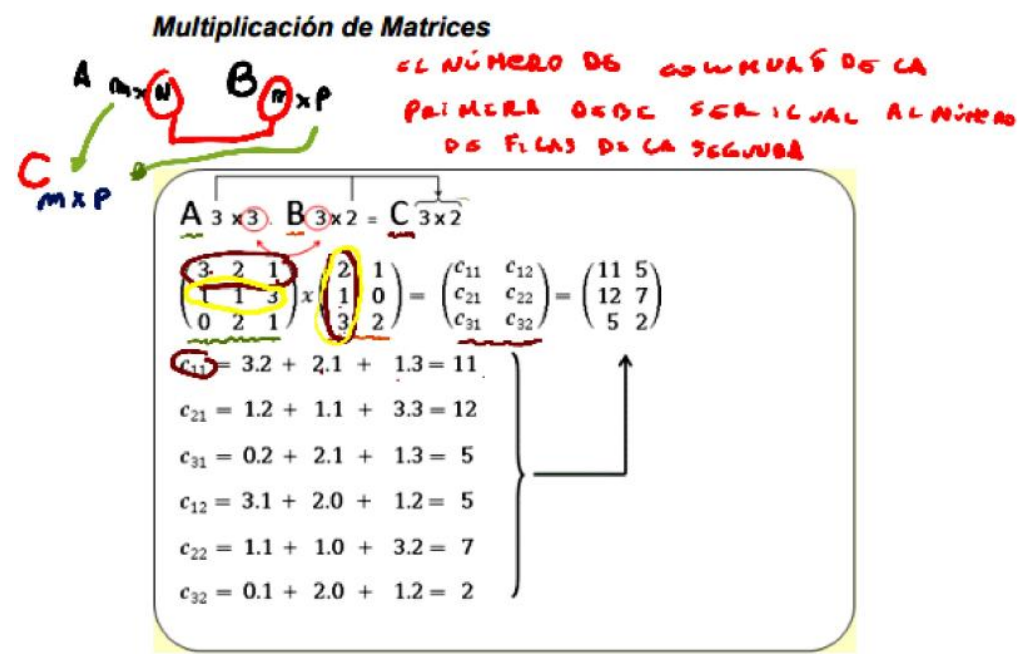

Figura 3. Ejemplo de uso de la tableta gráfica. 
Además, la tableta gráfica permite escribir sobre apuntes digitales (Figura 3), lo cual no es posible en una pizarra convencional. Incluso existen software que cuentan con un fondo cuadriculado o con los ejes cartesianos, de forma que permiten graficar funciones o trabajar los temas de geometría.

La desventaja de esta herramienta es el costo y que el profesorado debe acostumbrarse a escribir sobre la tableta, de forma que tiene que dimensionar el tamaño de la letra, ya que se proyecta directamente en la pantalla.

\section{Software GeoGebra}

La utilización de este software libre estuvo acompañada de las explicaciones del profesorado, aunque también fue utilizado de forma autónoma por el estudiantado (Del Río et al., 2020). En el primer caso, el profesorado utilizó la herramienta para graficar las funciones (Figura 4) y analizarlas: dominio, ordenada al origen, raíces, etc.

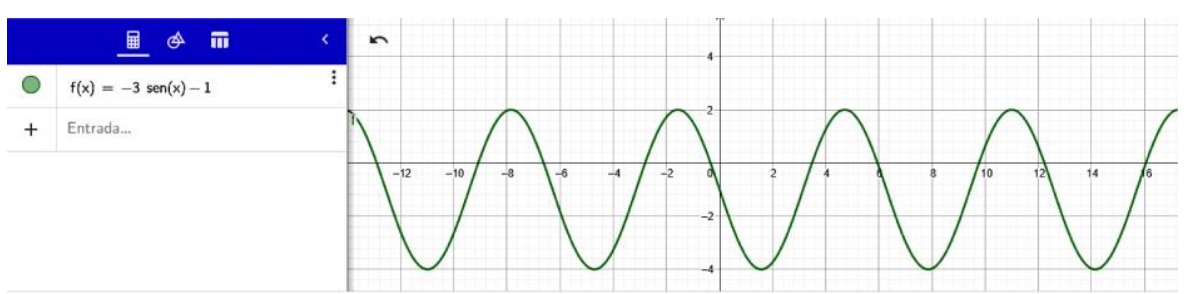

Figura 4. Gráfico de función trigonométrica producido mediante GeoGebra.

En el segundo caso, se sugería la utilización del software para generar una autocorrección de algunas actividades, tales como ecuaciones, límites, derivadas, integralesyoperaciones con matrices.

\section{Segunda pregunta formulada}

En relación con el segundo punto, el enfoque CTS en los perfiles de ingeniería y, en este caso, en el fortalecimiento del perfil de la microbiología clínica e industrial, permitiría contribuir a la formación matemática sistematizada a partir de la solución de problemas profesionales. Esto tiene como propósito promover el desarrollo económico, social y cultural, como una unidad entre la abstracción matemática científica y la matemática generativa (Faustino et al., 2019). Estos autores resumen, en una figura, el enfoque CTS (Figura 5).

Siguiendo al artículo citado, se introdujeron situaciones problemáticas sobre epidemias y pandemias, a partir de los contenidos mínimos de los cursos, trabajando temas de límite y de función exponencial para analizar la evolución de una enfermedad. A modo de ejemplo, se menciona una actividad de Matemática II que consistía en el análisis de una función, $\mathrm{f}(\mathrm{t})$, que representaba la cantidad de personas que contraían una enfermedad en un tiempo t. A partir de allí, se preguntaba cuál era la información que indicaba el límite cuando t tiende a infinito, cuántas personas se contagiaron inicialmente y se solicitaba un gráfico aproximado de la curva de la enfermedad, utilizando el software GeoGebra. De esta forma, esta actividad permitía trabajar conceptos de análisis matemático y de la vida cotidiana (Tabla 2). 


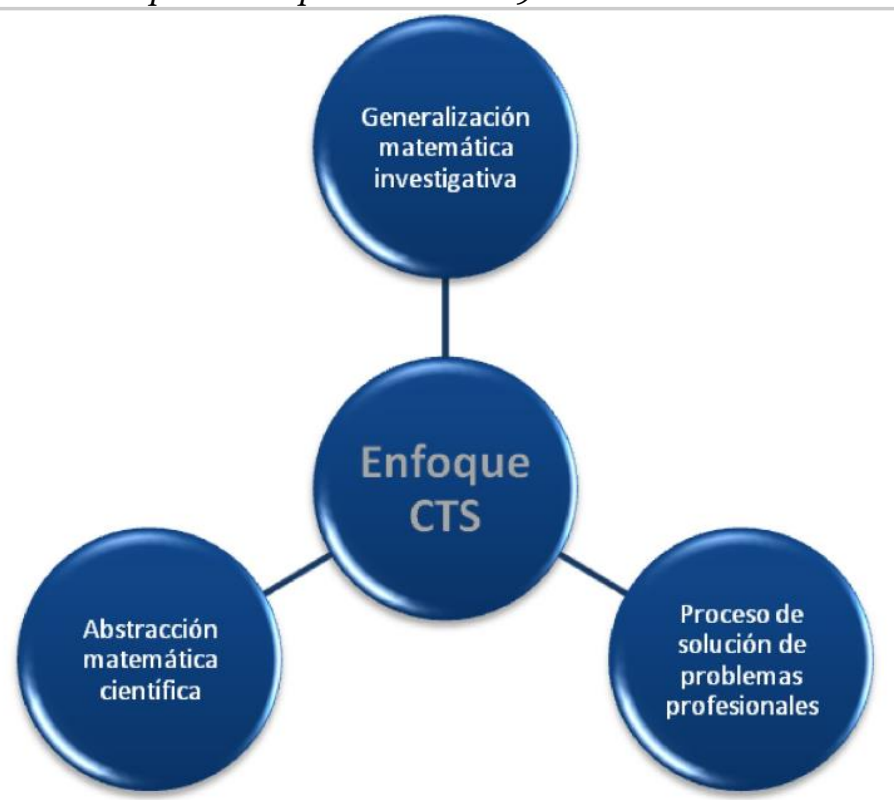

Figura 5: Formación matemática sistematizada a partir del enfoque Ciencia, Tecnología, Sociedad. Extraído de Faustino et al. (2019).

\begin{tabular}{cc}
\hline Temas de análisis matemático & Temas de la vida cotidiana \\
\hline - Funciones: dominio e imagen & - Evolución de una enfermedad \\
- Gráfico de funciones & - Interpretación de curvas \\
- Límites & \\
- Interpretación de límite & \\
- Modelos matemáticos & \\
\hline
\end{tabular}

Tabla 2: Temas CTS de trabajo en Matemática II.

Otro método aplicado y discutido en el análisis de la situación epidemiológica fue el modelo SIR (Susceptibles, Infectados, Recuperados), que se utiliza para el conocimiento de epidemias (Amster, 2020). Este modelo se utilizó dentro del eje de aplicaciones de la matemática a la microbiología clínica.

Como se mencionó anteriormente, los temas CTS incluyen elaborar temas de "Naturaleza de la Ciencia y la Tecnología". Por este motivo, en Matemática I se desarrollaron aspectos de historia de la matemática, por ejemplo, el surgimiento de los números complejos a partir de la vida de Rafael Bombelli (Bagni, 2001).

Tercera pregunta formulada

Finalmente, en relación con la tercera pregunta, que hace referencia a los resultados obtenidos, se observó que se registraron 41 estudiantes para cursar Matemática I. De ellos, 28 aprobaron por promoción, 2 deben rendir la evaluación final integradora (EFI) y 11 dejaron de cursar (abandonaron). En relación con Matemática II, los 10 estudiantes inscriptos aprobaron por promoción. 


\section{Discusión y conclusiones}

La situación de pandemia ha modificado la enseñanza de las ciencias en todos los niveles educativos. Los recursos tecnológicos implementados podrían ser de utilidad para el regreso a la presencialidad en un futuro. Asimismo, permiten el abordaje de la matemática desde otra perspectiva y también desarrollar otras competencias en el estudiantado: autocrítica, autocorrección y trabajo colaborativo. Asimismo, la utilización de las diferentes herramientas posiciona a la enseñanza de la matemática en un aspecto innovador, pues se podría dedicar el tiempo que antes se utilizaba en la construcción de gráficos de forma manual, a la búsqueda de aplicaciones de los contenidos abordados.

En relación con la enseñanza CTS, la misma permite trabajar aspectos de la vida cotidiana a partir de los contenidos disciplinares. De esta forma, para un perfil de graduados de Microbiología, se fomenta una "Matemática para no matemáticos", de forma de contextualizar su enseñanza. Cabe mencionar que este tipo de abordaje permite trabajar de forma multidisciplinaria con otras asignaturas como Genética y Química. Algunos ejemplos podrían ser la utilización de problemas con logaritmos para el cálculo del $\mathrm{pH}$ y el análisis de curvas de desarrollo de microorganismos. Este tipo de prácticas invita a pensar un abordaje transversal entre las diferentes asignaturas de primer y segundo año. Asimismo, el enfoque CTS permite la inclusión de contenidos de NdCyT, como se mencionó anteriormente. De esta forma, siguiendo la categoría utilizada por Vázquez Alonso (2014), las prácticas realizadas en el curso corresponderían a los siguientes temas de NdCyT (Tabla 3):

\begin{tabular}{|c|c|c|}
\hline Categorías & Aspectos & $\begin{array}{l}\text { Tema abordado en } \\
\text { matemática }\end{array}$ \\
\hline $\begin{array}{l}\text { Sociología externa } \\
\text { de la ciencia }\end{array}$ & $\begin{array}{l}\text { - Influencia de la sociedad sobre la } \\
\text { política de CyT } \\
\text { - Influencia de la ciencia y } \\
\text { tecnología sobre la sociedad. } \\
\text { Influencia de la ciencia escolar } \\
\text { sobre la sociedad } \\
\text { - Controversias socio-científicas }\end{array}$ & $\begin{array}{l}\text { Aplicación de las funcio- } \\
\text { nes en el análisis de } \\
\text { enfermedades }\end{array}$ \\
\hline $\begin{array}{l}\text { Sociología interna } \\
\text { de la ciencia }\end{array}$ & $\begin{array}{l}\text { - Característica de los científicos } \\
\text { - Construcción social del conoci- } \\
\text { miento } \\
\text { - Construcción social de la } \\
\text { tecnología }\end{array}$ & $\begin{array}{l}\text { Motivación, capacidades y } \\
\text { competencias en matemá- } \\
\text { tica }\end{array}$ \\
\hline Epistemología & $\begin{array}{l}\text { - Naturaleza del conocimiento } \\
\text { científico }\end{array}$ & $\begin{array}{l}\text { Historia de los números. } \\
\text { Principales investigadores } \\
\text { de la matemática }\end{array}$ \\
\hline
\end{tabular}

Tabla 3: Taxonomía de NdCyT y su aplicación en matemática.

Por otro lado, el porcentaje de aprobación por promoción muestra que este enfoque y los recursos utilizados para la enseñanza de la matemática permiten asegurar la permanencia del estudiantado en los cursos y esto permite abrir nuevos caminos de investigación sobre la temática. 
Proyecciones de la investigación

Como perspectiva a futuro, se continuará con la implementación de este tipo de prácticas, con el fin de acercar la matemática a los aspectos aplicados de la microbiología clínica a industrial, a partir de una propuesta de abordaje de alguna zoonosis y su análisis. Por ejemplo, para el caso de matemática I, se proyecta relacionar la enfermedad con la curva de crecimiento de contagios, la gráfica y el análisis de la función (utilizando software específico). Para el caso de Matemática II, se utilizará el mismo eje, pero enfocándose en la aplicación de límite, en la predicción del comportamiento de alguna enfermedad y en el uso de ejemplos y modelos de la topología en epidemias y en el proceso de vacunación (Grima \& Borja, 2017). Para ello, se utilizará una metodología cuasi experimental de pre y post análisis, utilizando las siguientes herramientas:

- El Cuestionario de Opiniones sobre Ciencia Tecnología y Sociedad (COCTS) (Manassero et al., 2003). Este cuestionario está formado por 100 cuestiones para evaluar un rasgo concreto de Naturaleza de la Ciencia. El COCTS proporciona, para un determinado ítem, varias frases de juicios, en las que la persona encuestada valora su grado de acuerdo con cada frase.

- El test de Halpern (Halpern, 2006), para evaluar las destrezas de Pensamiento Crítico del estudiantado.

Asimismo, se prevé realizar un análisis comparativo entre los años 2020 y 2021, en relación con el porcentaje del estudiantado aprobado y desaprobado, como así también, se indagará, mediante encuestas y entrevistas, la visión del estudiantado sobre el uso delos recursos tecnológicos y el enfoque CTS.

\section{Declaración de conflictos de intereses}

No existe conflicto de intereses.

\section{Agradecimientos}

A la Facultad de Ciencias Veterinarias y en especial, a la dirección de la carrera de Microbiología Clínica e Industrial, al departamento de Ciencias Básicas y al equipo de docentes y adscriptos de los cursos de Matemática.

\section{Bibliografía}

Álvarez M, Murúa R. 2020. Interpretación de gráficos: el uso de GeoGebra. Revista de Educación Matemática, 35(3):7-19. https://doi.org/10.33044/revem.31160

Amster P. 2020. La matemática de las epidemias. Revista de Educación Matemática, 35(2):5-20.

Ascheri M, Pizarro RA. 2006. Uso de tecnología en la enseñanza-aprendizaje de temas de cálculo numérico. Acta Latinoamericana de Matemática Educativa. 19:879-885.

Bagni GT. 2001. La introducción de la historia de las matemáticas en la enseñanza de los números complejos. Una investigación experimental en la educación media superior. Revista Latinoamericana de Investigación en Matemática Educativa. 4(1):45-61.

Bonservizi VM, Sgreccia NF. 2021. Articulación de las tecnologías a través de la carrera Profesorado en Matemática de la Universidad Nacional de Rosario. Educação Matemática Debate. 5(11):10. 
Baumann AJ. 2021. Cambios en la estrategia de enseñanza. Aplicación del aprendizaje basado en problemas ante la pandemia. Educación en la Química, 27(1):110-114.

Cabezas Rosa L. 2020. Propuesta didáctica de matemáticas durante la pandemia del COVID 19. Tesis de Maestría.Carrera Universidad de Almería.

http://repositorio.ual.es/handle/10835/10080

Carp DJ, Dillon LG, Porro S. 2016. Las mujeres y la química. Indagatio Didactica. 8(1):1838-1849. https://doi.org/10.34624/id.v8i1.12083

Del Río LS, Knopoff PA, Boero E, Ciliberti LF. 2020. Innovación e improvisación en el marco de la pandemia de COVID-19: relato de una experiencia. Trayectorias Universitarias 6(10):1-10. https://doi.org/10.24215/246 90090 oe20

Diaz Dávila LC, Britos JD, Hirschfeld G, Comerci S, Galoppo JL, Martiarena N. 2020. La pandemia. Acciones para facilitar el aprendizaje en Matemática durante el ingreso a carreras de Ingeniería. En: Libro de Actas del XV Congreso Nacional de Tecnología en Educación y Educación en Tecnología (TE\&ET 2020). pp56-65.

http://sedici.unlp.edu.ar/handle/10915/103576

Di Domenicantonio R, Langoni L. 2021. Coordinación de materias masivas de Matemática en la Facultad de Ingeniería de la UNLP durante la pandemia COVID-19. TE \& ET: Revista Iberoamericana de Tecnología en Educación y Educación en Tecnología. 28:168-73.

Facultad de Ciencias Veterinarias, Universidad Nacional de La Plata. 2013. Ordenanza 282 de la Carrera de Microbiología Clínica e Industrial.

Faustino A, Pérez Sánchez N, Diéguez Batista R. 2019. Formación matemática sistematizada a partir del enfoque ciencia, tecnología y sociedad, en el perfil ingenieril. Revista Educación, 43(1):73-97.

https://doi.org/10.1551 7/revedu.v43i1.28233

García-Planas MI, Torres JT. 2021. Transición de la docencia presencial a la no presencial en la UPC durante la pandemia del COVID-19. International Journal of Educational Research and Innovation. 15:177-87. https:// doi.org/10.46661/ijeri.5015

Grima CI, Borja EF. 2017. Las matemáticas vigilan tu salud: modelos sobre epidemias y vacunas. NextDoorPublishers. Colección Café Cajal.

Halpern DF. 2006. Is intelligence critical thinking? Why we need a new construct definition for intelligence. En: P. Kyllonen, I. Stankov, R.D. Roberts (Eds.). Extending intelligence: Enhancement and new constructs. Mahwah, NJ: Erlbaum Associates.

Hidalgo-Moncada D, Díez-Palomar J, Vanegas Muñoz Y. 2020. Formación de maestros de educación primaria en el contexto de confinamiento: la importancia del aprendizaje autorregulado en las matemáticas. Revista miscelánea de investigación. 32(1):40-48.

Lampert D, Larsen A, Unzaga J, Mórtola E, Porro S. 2020. La enseñanza de temas de naturaleza de la ciencia y la tecnología y el desarrollo del pensamiento crítico en inmunología veterinaria: un estudio de caso. Revista de Medicina Veterinaria (en línea). 101(1):57.

Lampert D, Porro S. 2019. Innovations in the development of critical thinking and the teaching of the nature of science and technology: background and proposal for food engineering course of studies. En: HEAD'19. 5th International Conference on Higher Education Advances. Editorial Universitat Politècnica de València. pp. 261-269.

Lescano A, Puy J, Puy A. 2021. De la presencialidad a la virtualidad: enseñar matemáticas en pandemia. TE \& ET: Revista Iberoamericana de Tecnología en Educación y Educación en Tecnología No 28 - Especial, 294-302.

Manassero MA, Vázquez-Álonsa Á, Acevedo-Díaz JA. 2003. Cuestionario de opiniones sobre ciencia, tecnología y sociedad (COCTS). Princeton, NJ: Educational Testing Service.

Ortiz-Buitrago J, Sánchez Tovar L. 2020. Educación en tiempos de incertidumbre. Una mirada a la actuación del docente de matemáticas. Matemáticas, Educación y 
Sociedad. 3(3):29-43.

Porro S, Roncaglia DI. 2016. La educación CTS en la formación de docentes y otras profesiones. Indagatio Didactica. 8(1):61-73.

https://doi.org/10.34624/id.v8i1.3094

Rojas Maldonado ER, Toscano Galeana J. 2021. Instrumento para evaluar los conocimientos matemáticos previos para la enseñanza del concepto de límite durante la pandemia SARS-CoV-2. RIDE Revista Iberoamericana para la Investigación y el Desarrollo Educativo. 11(22):e235. https://doi.org/10.23913/ride.v11i22.953

Sánchez Pachas CI. 2020. Herramientas tecnológicas en la enseñanza de las matemáticas durante la pandemia COVID-19. HAMUT'AY. 7(2):46-57.

https://doi.org/10.21503/hamu.v7i2.2132

Tenreiro-Vieira C, Vieira RM. 2016. Educação em ciências e matemática com orientação CTS promotora do pensamento crítico. CTS: Revista Iberoamericana de Ciencia, Tecnología y Sociedad. 11(33):143-159.

Valarezo Neira DR. 2021. Influencia del uso de las herramientas virtuales en la enseñanza aprendizaje de las matemáticas. Bachelor's thesis, Machala: Universidad Técnica de Machala. http://186.3.32.121/handle/4800 o/16315

Vázquez Alonso Á. 2014. Enseñanza, Aprendizaje y Evaluación en la Formación de Docentes en Educación CTS en el contexto del siglo XXI. Uni-pluri/versidad. 14(2):37-49.

Zenteno-Mendoza BE, Garritz A. 2010. Secuencias dialógicas, la dimensión CTS y asuntos socio-científicos en la enseñanza de la química. Revista Eureka sobre Enseñanza y Divulgación de las Ciencias. 2-25.

https://doi.org/ 10.25267/Rev_Eureka_ensen_divulg_cienc.2010.v7.i1.01 